\title{
Returned Amount
}

National Cancer Institute

\section{Source}

National Cancer Institute. Returned Amount. NCI Thesaurus. Code C78722.

The quantity of a product that has been returned. 